\title{
The Importance of Histochemical Detection of Oxidative Damage in Tissue
}

\author{
M.A. Smith, * X. Zhu, * H. Lee, ${ }^{*}$ R.J. Castellani** and G. Perry***
}

*Department of Pathology, Case Western Reserve University, Cleveland, Ohio 44106

** Department of Pathology, University of Maryland, Baltimore, Maryland, 21201

*** College of Sciences, University of Texas at San Antonio, San Antonio, Texas 78249

Oxidative stress is a key component involved in the progression of numerous diseases and it is primarily through novel histological and microscopic techniques that it is now clearly established that free radical oxidative damage mainly of lipids, proteins, nucleic acids and sugars can be used to identify the hallmark signature of "oxidative stress" within tissues. Our research in this area has focused on the two principal pathways for protein modification, one involving hemolytic hydroxyl radical-like chemistry that results in protein-based carbonyls, and the other involving electrophilic nitration of vulnerable side chains. In the presence of buffering bicarbonate, peroxynitrite forms a $\mathrm{CO}_{2}$ adduct which augments its reactivity. Formation of 3-nitrotyrosine by this route has become the classical protein marker for the presence of peroxynitrite [1]. Protein-based carbonyls can be detected by two methods, either derivatization with 2,4-dinitrophenylhydrazine (DNP-H) followed by detection of the protein-bound hydrazones using an enzyme-linked anti-2,4dinitrophenylhydrazone antibody or derivatization with biotin-hydrazide followed by the detection of the protein-bound acyl hydrazone with enzyme-linked-avidin or strepavidin [2,3]. Glycation of proteins by reducing sugars (Maillard reaction) results in a profile of time-dependent adduct evolution that becomes especially susceptible to oxidative amplification. In addition, oxidative stress can result in oxidized sugar derivatives which can then modify proteins through a process known as glycoxidation. Also, oxidative stress results in lipid peroxidation and the production of a range of electrophilic and bifunctional aldehydes that modify a large variety of proteins. The most important protein modifications are referred to as advanced glycation end products (AGEs) and advanced lipoxidation end products (ALEs). Protein modifications can result in both non-crosslinked and crosslinked AGEs and ALEs, the latter arising from the potential bifunctional reactivity of such lipid-derived modifiers 4-hydroxy-2-nonenal (HNE) and malondialdehyde (MDA) [4]. Oxidative damage to nucleic acids is also involved in oxidative stress within tissues resulting in base modifications, substitutions and deletions. The most common modification studied is 8hydroxyguanosine $(8 \mathrm{OHG})$ which is considered a signature of oxidative damage to nucleic acids. Obviously oxidative stress plays an important role in the pathogenesis of disease states and warrants considerable attention [5].

References

[1] M.A. Smith et al., Proc. Natl. Acad. Sci. U S A 91 (1994) 5710-5714.

[2] L.M. Sayre, G. Perry, M.A. Smith, Methods Enzymol. 309 (1999) 133-152.

[3] M.A. Smith et al., Nature 382 (1996) 120-121.

[4] PNAS AGE

[5] This research was supported by the National Institutes of Health and the Alzheimer's Association. 